Intervenção em Saúde do Trabalhador

Heloisa Aparecida Souza ${ }^{a}$ (iD) http://orcid.org/0000-0002-0297-0858

Marcia Hespanhol Bernardo ${ }^{a}$

(iD) https://orcid.org/0000-0002-8068-6238

a Pontifícia Universidade Católica de Campinas, Faculdade de Psicologia. Campinas, SP, Brasil.

Contato:

Heloisa Aparecida Souza

E-mail:

heloisa_apsouza@yahoo.com.br

Artigo com base na tese de doutorado intitulada Saúde mental relacionada ao trabalho na rede pública de saúde brasileira: concepções e atuações transformadoras, de Heloisa Aparecida Souza, defendida em 2017 no

Programa de Pós-Graduação em Psicologia da Pontifícia Universidade Católica de Campinas.

As autoras declaram que o estudo foi subvencionado pelo Programa de Suporte à Pós-Graduação de Instituiç̃oes Particulares da Coordenação de Aperfeiçoamento de Pessoal de Nível Superior (Prosup/ Capes) concedido em março de 2013, e que não há conflitos de interesses.

As autoras informam que o trabalho não foi apresentado em evento científico.

\section{Prevenção de adoecimento mental relacionado ao trabalho: a práxis de profissionais do Sistema Único de Saúde comprometidos com a saúde do trabalhador}

\author{
Work-related mental illness prevention: the praxis of Brazilian \\ public health system professionals committed to the \\ worker's health
}

\section{Resumo}

Introdução: apesar das inúmeras evidências de que as condições de trabalho na atualidade geram adoecimento mental nos trabalhadores, as políticas públicas que abordam essa temática ainda são frágeis no Brasil. Objetivos: identificar e analisar a práxis de profissionais da área da saúde pública que compreendem a complexidade presente no processo de saúde-adoecimento mental, considerando os aspectos relacionados às situações de trabalho. Métodos: pesquisa qualitativa, com uso de entrevistas abertas realizadas com nove profissionais que atuavam na Atenção Básica, em Centro de Atenção Psicossocial (Caps) e em Centros de Referência em Saúde do Trabalhador (Cerest) do estado de São Paulo. Resultados: as práticas dos profissionais vão do atendimento aos trabalhadores já adoecidos a propostas de promoção de saúde psíquica nos ambientes de trabalho. Os profissionais revelaram compreensão da relação entre saúde mental e trabalho e demonstraram comprometimento ético e político com a temática e propostas do campo da Saúde do Trabalhador. Conclusão: para que ações como as identificadas aconteçam com maior frequência, é necessário que se invista na formação crítica dos profissionais da saúde, no protagonismo da classe trabalhadora e que se consolidem e disseminem políticas públicas que visem às ações de prevenção do adoecimento mental relacionado ao trabalho.

Palavras-chave: saúde mental; trabalho; práxis; Sistema Único de Saúde (SUS); profissionais de saúde.

\begin{abstract}
Introduction: despite the innumerable evidences that the current working conditions generate mental illness in workers, public policies that approach this subject are still fragile in Brazil. Objectives: to identify and analyze the praxis of public health professionals who understand the complexity of the work-related mental health-illness process. Methods: a qualitative study was carried out using the open interview technique with nine health professionals from Psychosocial Care Centers (Caps) and Occupational Health Reference Centers (Cerest) of the São Paulo State primary care network. Results: the professionals' practices involve caring to workers who were already ill and proposals for psychic health promotion in the work environment. Their praxis revealed understanding of the relationship between mental health and work, and demonstrated their ethical and political commitment to the theme and to the Occupational Health field proposals. Conclusion: for the identified actions to be taken more frequently it is necessary to invest in health professionals' argumentative trainings, to recognize the working class central role, and to consolidate and disseminate public policies aimed at preventing work-related mental illness.
\end{abstract}

Keywords: mental health; work; praxis; Brazilian unified health system (SUS); health professionals.
Revisado: 04/09/2018

Aprovado: 17/09/2018 


\section{Introdução}

Depressão, tentativas de suicídios, uso abusivo de álcool e drogas, estresse, crises de ansiedade, fadiga e esgotamento profissional estão se tornando cada vez mais comuns e há muitas evidências da relação entre essas expressões do sofrimento humano e as formas de organização do trabalho existentes na atualidade.

De acordo com a Organização para a Cooperação e Desenvolvimento Econômico (OECD) ${ }^{1}$, as doenças mentais são responsáveis por uma redução significativa do potencial de oferta de trabalho, pelo aumento do absenteísmo e pela exclusão social de milhares de pessoas, gerando altos custos econômicos e sociais. A Organização Mundial da Saúde (OMS) e a Organização Internacional do Trabalho (OIT) afirmam que mais de $30 \%$ dos trabalhadores dos países industrializados sofrem com algum tipo de transtorno mental ${ }^{2}$. No Brasil, os dados do Ministério da Previdência e Assistência Social (MPS) revelam a alta incidência de doenças psíquicas entre a população em idade produtiva, representando a terceira maior causa de afastamento do trabalho no país ${ }^{3}$.

Para Seligmann-Silva ${ }^{4}$, Bernardo ${ }^{5}$, Praun $^{6}$, Gaulejac $^{7}$, Pina e Stotz ${ }^{8}$, entre outros autores, a falta de enfrentamento das situações de trabalho que geram um processo de adoecimento físico e mental nos trabalhadores está relacionada com as atuais formas de organização financeira e produtiva, a implementação de novos mecanismos de gestão e a maneira como as avançadas tecnologias são utilizadas. Tais autores afirmam que o trabalho na atualidade passou a exigir um maior envolvimento da subjetividade dos trabalhadores. Nesse contexto, o cotidiano laboral vem se caracterizando pela contradição entre discursos gerenciais bastante sedutores e práticas extremamente exigentes que tendem a enfraquecer a organização coletiva.

Dessa forma, o problema é naturalizado e negligenciado, havendo poucas iniciativas para a defesa da saúde mental da classe trabalhadora. Parece haver relutância por parte do poder público, dos administradores das empresas e dos próprios trabalhadores em considerar os contextos macros e microssociais, e assumir que o trabalho pode agir negativamente sobre o psiquismo de quem trabalha. Bernardo e Garbin $^{9}$ nos ajudam a compreender o motivo dessa realidade ao afirmarem que, em nossa sociedade, permanece a ideia de que o trabalho possui uma conotação positiva e o sofrimento/adoecimento psíquico, em geral, é visto como um sinal de fraqueza individual. As autoras discutem ainda a importância da atenção à saúde mental relacionada ao trabalho no Sistema Único de Saúde (SUS), o qual também enfrenta inúmeros obstáculos e desafios ${ }^{9,10}$.
Em uma realidade de contradições e dificuldades, em geral, o enfrentamento dos agravos à saúde mental relacionados ao trabalho é bastante frágil. Entretanto, existem muitos profissionais da saúde que têm buscado associar seus saberes com os dos trabalhadores, para realizar ações críticas e contextualizadas, agindo de forma a atender as demandas de adoecimento psíquico relacionado ao trabalho. Assim, o objetivo deste artigo é identificar e analisar a práxis de profissionais da saúde pública que compreendem a complexidade presente no processo de saúde-doença mental, considerando os aspectos relacionados às situações laborais. Vale destacar que práxis é compreendida neste trabalho como a ação criativa, consciente e transformadora da realidade e do ser humano ${ }^{11}$.

\section{Métodos}

\section{Referencial teórico metodológico}

O presente trabalho é baseado em pesquisa empírica de natureza qualitativa. Segundo Minayo ${ }^{12}$, esse tipo de estudo considera aspectos não mensuráveis, como aspirações, motivos, crenças, atitudes e valores na busca por significados, percebendo o dinamismo da realidade econômica, histórica e cultural, possibilitando, assim, um enfrentamento mais adequado das restrições impostas pelo contexto social.

Adotamos o referencial teórico do Desgaste Mental, que, segundo Seligmann-Silva ${ }^{4}$, seria uma abordagem integradora de importantes concepções teóricas, como a Psicodinâmica do Trabalho e a Teoria do Estresse, entre outras. Tendo o materialismo histórico dialético como base, esse enfoque reconhece a efetiva participação do contexto social e de trabalho no processo saúde-doença mental dos trabalhadores. Nesse sentido, ações criativas e transformadoras de prevenção em saúde do trabalhador e, mais especificamente, em Saúde Mental Relacionada ao Trabalho (SMRT) devem levar em consideração a vivência e os saberes dos trabalhadores ${ }^{13}$, contribuindo para que os diferentes atores sociais possam participar ativamente da superação da visão fatalista que é predominante.

Para Martín-Baró ${ }^{14}$, o fatalismo pode ser considerado o responsável pelo conformismo e pela falta de mobilização dos indivíduos. O autor sugere que a visão fatalista é fruto de um sistema de ideologias orquestrado para atribuir a responsabilidade pela marginalização e pela exclusão às vítimas, limitando seu poder de reação e de enfrentamento das mazelas impostas pelos sistemas social e econômico vigentes. Dessa maneira, podemos considerar que as ações que têm o intuito de favorecer 
o desenvolvimento da consciência da população sobre a relação entre sofrimento/adoecimento mental e as situações de trabalho colaboram com a ruptura do esquema ideológico do fatalismo, beneficiando uma postura mais ativa em busca de melhores condições de trabalho, diminuindo, desta forma, a culpabilização das vítimas e evitando a aceitação das realidades construídas socialmente como sendo condições naturais e imutáveis.

Conforme nos lembra Seligmann-Silva ${ }^{4}$, podemos encontrar com facilidade a exploração do fatalismo nas relações de trabalho. Essa exploração, segundo a autora, contribui com o fortalecimento e manipulação da ideia de que os riscos de acidentes e de adoecimento são inerentes ao trabalho e inevitáveis. Por ser naturalizado socialmente e ocorrer de forma insidiosa, progressiva e silenciosa, raramente, o desgaste e o adoecimento mental do trabalhador são relacionados com as situações de trabalho vivenciadas coletivamente.

Entendemos que as práxis aqui discutidas podem ser consideradas exitosas e exemplares, revelando que, indiferentemente da área de formação ou do campo de atuação, o profissional do SUS pode lançar um olhar atento e oferecer grandes contribuições na atenção e prevenção do adoecimento mental relacionado ao trabalho, rompendo com as concepções individualizantes, naturalizantes e fatalistas que predominam na compreensão do processo saúde-doença mental no ambiente de trabalho e incentivando o protagonismo da classe trabalhadora.

\section{Participantes, levantamento e análise de informações}

Com o objetivo de acessar profissionais com atuações que servissem de modelo no campo da SMRT nos diversos equipamentos que compõem o SUS, elegeu-se os participantes de modo intencional. De acordo com Duarte ${ }^{15}$, essa escolha intencional permite ao pesquisador selecionar sujeitos que demonstram conhecimento e envolvimento com o tema pesquisado. A aproximação com os participantes ocorreu por meio da rede de contato das pesquisadoras e/ou indicações de profissionais que apresentassem trajetórias de práticas voltadas para a defesa da saúde mental dos trabalhadores.

No segundo semestre de 2015, foram realizadas entrevistas abertas com nove profissionais, sendo três psicólogos, uma médica, uma terapeuta ocupacional, uma fisioterapeuta, uma auxiliar de enfermagem e duas enfermeiras. Esses profissionais atuavam na Atenção Básica, em Centro de Atenção Psicossocial (Caps) e em Centros de Referência em Saúde do Trabalhador (Cerest) da capital e de outras cinco cidades do interior do estado de São Paulo:
Campinas, Marília, São Bernardo do Campo, São José dos Campos e Valinhos.

Após o projeto de pesquisa ser aprovado pelo Comitê de Ética em Pesquisa da Pontifícia Universidade Católica de Campinas, com o parecer $\mathrm{n}^{\mathrm{o}}$ 914.673, de 8 de dezembro de 2014, foram realizadas as entrevistas, nas quais era solicitado que os participantes falassem detalhadamente sobre suas concepções e experiências profissionais que envolvessem as questões da saúde mental dos trabalhadores. As entrevistas foram gravadas, transcritas e, posteriormente, analisadas de acordo com a perspectiva proposta por Minayo ${ }^{12}$, buscando o entendimento crítico dos depoimentos como resultados de processos sociais que são frutos de múltiplas determinações e procurando revelar o engajamento dos entrevistados com a atenção à saúde mental dos trabalhadores.

\section{Resultados e discussão}

Os resultados desta pesquisa são divididos em duas seções. Na primeira, abordamos concepções dos profissionais sobre a relação entre trabalho e saúde mental e, em seguida, focalizamos as principais práticas adotadas por eles na atenção e na promoção da saúde mental dos trabalhadores.

\section{Concepções sobre a relação entre trabalho e adoeci- mento mental}

Para a realização de ações que se configurem como práxis é necessário que quem as faça tenha consciência sobre o contexto social, a temática trabalhada e seu papel na sociedade ${ }^{11}$. É por essa razão que, antes de abordar as práticas dos participantes da pesquisa, apresentamos algumas de suas concepções a respeito da sociedade contemporânea, do mundo do trabalho e da SMRT. Consideramos que, de certa forma, essas compreensões demarcam o posicionamento ético e político dos participantes e balizam suas práticas na área da saúde.

Um primeiro aspecto a ser destacado é que as concepções dos participantes da pesquisa se distanciam da postura fatalista e individualizante, reconhecendo elementos do trabalho que colaboram com o processo saúde-doença mental. Neste sentido, a psicóloga A, que atua em um Centro de Referência em Saúde do Trabalhador, fez a seguinte observação:

Não podemos nos deter somente às características individuais, precisamos compreender as pessoas inseridas em um contexto social e de trabalho que padece e, na maioria das vezes, é muito exigente, injusto e impiedoso. Estamos vivendo uma epidemia de adoecimento mental. Não podemos achar isso normal, precisamos entender que as pessoas não 
estão suportando as tensões e pressões desse mundo enlouquecido.

A enfermeira A, que atua na Atenção Básica, fez a seguinte reflexão:

\begin{abstract}
Se a sociedade está perdendo os valores éticos e morais e se a precarização do trabalho está banalizada, precisamos nos esforçar para não aderir a essa onda e colaborar para amenizar os efeitos dessa realidade na vida e na saúde de nossos pacientes.
\end{abstract}

Sem explicitar referenciais teóricos, os participantes da pesquisa parecem construir seu olhar a partir de uma concepção ampliada, que, além das características individuais, envolve a compreensão de diferentes patamares da organização social e do trabalho, concepção que se aproxima do que é defendido por Seligmann-Silva ${ }^{4}$.

O estabelecimento de nexos entre agravos à saúde mental e as situações de trabalho existentes na atualidade costuma ser um grande desafio ao profissional da saúde ${ }^{4,16}$. As falas dos participantes indicam que procuram ir além do atendimento às demandas explícitas e tentam oferecer uma atenção integral aos trabalhadores/usuários, voltando-se para suas reais necessidades e para a compreensão das possíveis causas dos sofrimentos apresentados. A auxiliar de enfermagem, que também atua na Atenção Básica, por exemplo, falou sobre a percepção dos impactos na saúde mental nos trabalhadores que sofrem acidentes de trabalho:

\begin{abstract}
Acontece de eu estar fazendo um curativo nos ferimentos e os trabalhadores acidentados falarem sobre as humilhações que sofrem do chefe, do descaso da empresa, do medo de serem demitidos ao retornarem ao trabalho... Eles chegam com cortes, pancadas e até amputações e, enquanto atendemos esses trabalhadores, fica claro que, além das feridas do corpo, há feridas na alma dessas pessoas que podem ter sido provocadas pelos sonhos destruídos e pelas humilhações diárias.
\end{abstract}

Para a terapeuta ocupacional, que tem experiência na Atenção Básica e nos campos da Saúde do Trabalhador e da Saúde Mental, a história de trabalho do indivíduo adoecido mentalmente é essencial para a compreensão do quadro clínico e para a continuidade do tratamento. Em sua concepção, é um

desafio mudar o modelo de ambulatório para um modelo de atenção psicossocial. Se queremos um atendimento psicossocial de verdade, precisamos considerar a condição de vida e de trabalho das pessoas.

Ainda neste sentido, a fisioterapeuta, que atua na Atenção Básica, abordou a diferença entre os tipos de benefícios previdenciários que preveem mais direitos aos trabalhadores que apresentam adoecimento relacionado ao trabalho, afirmando que o estabelecimento da relação entre o trabalho e o adoecimento mental possui repercussão

tanto de caráter clínico - pois a postura do paciente é diferente quando reconhece que seu problema não é fruto de um fracasso seu e, sim, de um sistema que o explorou - como de caráter econômico e relativos aos direitos.

Ao abordarem os diversos aspectos envolvidos no processo de adoecimento mental dos trabalhadores, Maeno e Paparelli ${ }^{16}$ destacam a necessidade de ampliar a compreensão das possíveis causas, considerando também os "aspectos relacionados à convivência entre patrões e empregados, às hierarquias, ao ritmo, às formas de avaliação, à possibilidade de controle do trabalho, ou seja, à divisão do poder entre quem pensa e quem executa”. As concepções apresentadas pelos participantes demonstram que percebem esses elementos presentes no mundo de trabalho ao considerarem o processo de adoecimento da classe trabalhadora, além de revelarem a perseverança e engajamento ético e político com uma visão de saúde integral, considerando o contexto histórico e social. É a partir dessa perspectiva que pautam suas práxis no SUS, algumas das quais serão apresentadas a seguir.

\section{Práxis que estimulam a resistência dos trabalhadores e a preservação da saúde mental}

Os participantes relataram diversas ações que visam à saúde mental da classe trabalhadora, que vão do atendimento aos trabalhadores já adoecidos a propostas de promoção de saúde psíquica nos ambientes de trabalho. Aqui, será dado maior destaque para as práticas que procuram favorecer o fortalecimento dos trabalhadores, para que eles possam desconstruir a ideia fatalista do sofrimento/ adoecimento mental relacionado ao trabalho e possam promover a resistência aos elementos presentes no ambiente de trabalho que colaboram com a perda da saúde psíquica. Vale frisar que ações desse tipo são preconizadas desde a origem do SUS $^{17}$ e são consideradas primordiais no campo da Saúde do Trabalhador, muito embora se distanciem das práticas hegemônicas da área da saúde que, em geral, focalizam o atendimento clínico e individual ${ }^{4,9,18}$.

\section{Grupos psicossociais}

A maioria dos entrevistados acredita que os atendimentos em grupo estimulam os trabalhadores a discutir e compreender suas condições de vida, de trabalho e de saúde. Segundo alguns participantes, a realização de grupos evita o isolamento, proporciona o compartilhamento de experiências e potencializa o enfrentamento das dificuldades encontradas na realidade social e de trabalho - que, vale lembrar, 
na atualidade, incentiva a competição e o individualismo - e possibilita que os trabalhadores percebam como seus problemas se relacionam com o trabalho. A enfermeira B, profissional de um Centro de Atenção Psicossocial, destacou que:

A realização de grupos é uma importante ferramenta que temos. Através deles, os indivíduos se apoiam mutuamente, buscam respostas para seus problemas, refletem sobre suas vidas e tomam importantes decisões. Os profissionais de saúde são apenas facilitadores, os usuários são os protagonistas desses grupos que têm um grande potencial terapêutico.

Segundo Imbrizi, Keppler e Vilhanueva ${ }^{19}$, os grupos são importantes para problematizar o "sentimento de culpa e a adesão ao lugar de vítimas dos usuários que procuraram o serviço", favorecendo o "empoderamento dos trabalhadores” (p. 313). Neste sentido, a psicóloga A afirmou que o atendimento grupal oferecido pelos serviços públicos de saúde

favorece o autoconhecimento e a autoestima dos trabalhadores... No final, eles se descrevem mais confiantes, esperançosos e encorajados.

Os grupos a que se refere adquirem um caráter psicossocial, buscando estimular a autonomia e o fortalecimento coletivo dos trabalhadores.

Incentivo às associações e organizações coletivas dos trabalhadores

A médica entrevistada, que atua em um Cerest, abordou a importância de os profissionais da saúde apoiarem o desenvolvimento de associações que reivindiquem, na justiça, reparações dos danos provocados pelo trabalho à saúde e/ou à dignidade dos trabalhadores, considerando esses movimentos como uma interessante saída coletiva para amenizar as perdas vivenciadas nos ambientes de trabalho. Em sua concepção, essas organizações de trabalhadores, juntamente com os sindicatos, podem representar uma forma de impedir a impunidade de empresas que agem em detrimento da saúde dos trabalhadores:

\begin{abstract}
Podemos dar suporte emocional e técnico, auxiliando na compreensão, investigação e no rastreamento dos elementos do trabalho que prejudicam a saúde do trabaIhador. Teve um colega meu que desenvolveu um importantíssimo trabalho de bastidores, buscando nos detalhes formas concretas de provar o papel do trabalho no adoecimento dos trabalhadores expostos a substâncias químicas no local de trabalho, auxiliando muito para que uma associação se efetivasse e alcançasse seus objetivos.
\end{abstract}

Ainda no sentido do estímulo da organização coletiva, vários participantes da pesquisa abordaram a importância de favorecer modelos alternativos de organização do trabalho que não sejam pautados pela lógica competitiva e individualista, como a autogestão e as cooperativas de trabalhadores. A terapeuta ocupacional supervisionou diversas oficinas de trabalho e geração de renda nos moldes da economia solidária e destaca que, além de estimular a organização coletiva, essas atividades

podem significar a única fonte de rendas para alguns usuários.

A enfermeira B também destacou que a economia solidária pode ser um elemento essencial para o fortalecimento e a autonomia dos cidadãos que vivenciam sofrimento psíquico provocado ou agravado pelo trabalho, pois

a geração de renda alternativa diminui a exclusão social e a dependência financeira.

Dessa forma, a economia solidária pode representar um recurso terapêutico e um elemento essencial para o fortalecimento e a autonomia dos cidadãos que vivenciam sofrimento psíquico ${ }^{20}$.

\section{Vigilância em Saúde do Trabalhador}

As ações de Vigilância em Saúde do Trabalhador têm o objetivo de prevenir o adoecimento e promover a saúde, buscando no local de trabalho os elementos que podem prejudicar a saúde dos trabalhadores ${ }^{21}$. Os participantes que atuam em Cerest realizam esse tipo de ação e alguns elementos de suas falas revelaram que não se limitam ao papel de fiscais e autoridades sanitárias, tentando, constantemente, envolver os trabalhadores nas discussões e ações que inibam posturas abusivas das empresas.

O psicólogo B relatou que casos considerados "assédio moral" são frequentemente denunciados à área de vigilância do Cerest em que atua, abordando a dificuldade para realizar intervenções no local de trabalho e para observar essas condutas quando realizam ações de vigilância:

\begin{abstract}
Ao suspeitar que uma determinada empresa possua elementos nocivos à saúde mental de seus trabalhadores, emitimos uma notificação aos gestores. A empresa é forçada a assinar um termo no qual se compromete a realizar mudanças na organização de trabalho e a realizar campanhas informando os trabalhadores sobre o direito a condições dignas de trabalho e sobre a gravidade da prática de assédio moral. Na minha opinião, é fundamental informar os trabalhadores para que eles mesmos possam se organizar coletivamente para combater situações que coloquem sua saúde em risco.
\end{abstract}

A psicóloga C, gestora de um Cerest, narrou que, por meio de uma articulação com o Ministério Público do Trabalho, conseguiu fazer com que os valores de algumas penalidades aplicadas às empresas fossem revertidos diretamente para a realização de uma campanha de promoção da saúde nos locais 
de trabalho. Essa campanha atingiu muitos trabalhadores do município e teve o objetivo de informar a população sobre o direito ao trabalho digno e incentivar a denúncia de qualquer tipo de violência psicológica presenciada nos ambientes de trabalho.

Essas ações são importantes, visto que os tipos de organização do trabalho predominantes atualmente mesclam discursos sedutores - que apresentam a empresa como "amiga", "parceira", sendo uma "família" para o trabalhador - com práticas de gestão que se pautam na violência psicológica ${ }^{5}$. Daí a importância de favorecer que os trabalhadores tomem consciência do abismo existente entre os atraentes discursos e a realidade de violência à sua subjetividade que, muitas vezes, está presente nos locais de trabalho.

\section{Apoio matricial em Saúde do Trabalhador}

Os participantes da pesquisa demonstraram que valorizam também as ações denominadas apoio matricial ou matriciamento, para favorecer a conscientização dos demais profissionais do SUS sobre as possíveis relações do trabalho com o adoecimento mental. Desde o início dos anos 2000, as Políticas Nacionais de Saúde têm incentivado o apoio matricial como práticas que possuem o objetivo de proporcionar o alargamento do campo de atuação e a qualificação das ações das equipes da rede pública de saúde, por meio do suporte de profissionais de referência de diversas áreas especializadas ${ }^{22,23}$.

As atividades de matriciamento, no campo da Saúde do Trabalhador, podem ser consideradas como ações de educação permanente que facilitam "o compartilhamento de responsabilidades por todos os atores envolvidos no processo de cuidar da saúde dos trabalhadores" ${ }^{24}$. Essas atividades possuem também um papel importante na ampliação da autonomia e na reflexão dos próprios trabalhadores da saúde no processo de cogestão, podendo representar uma ferramenta de transformação tanto dos indivíduos quanto da realidade social ${ }^{25}$.

Para a enfermeira A o apoio matricial realizado pelo Cerest na Unidade Básica de Saúde em que atua contribui para a compreensão das necessidades dos usuários relacionadas ao mundo do trabalho. A terapeuta ocupacional também refletiu sobre a questão:

Todos os profissionais do SUS precisam ter em mente que o trabalho é algo central e representa muito mais do que uma linha a ser preenchida no formulário de acolhimento. Além de perguntar a ocupação do usuário, precisamos entender o que de fato a pessoa faz, como é sua atividade, como são as relações no local do trabalho e o matriciamento é muito importante para difundir essa concepção.

A psicóloga $\mathrm{C}$ listou as atividades de apoio matricial realizadas pelo Cerest em que atua. Essas ações tiveram o objetivo de sensibilizar os profissionais da área da saúde sobre a necessidade de incorporar a categoria trabalho na compreensão do processo saúde-doença mental dos trabalhadores. Entre as principais atividades realizadas, destacou os encontros com os psicólogos da Atenção Básica para refletir sobre a relação entre adoecimento mental e trabalho; reuniões com os diversos equipamentos públicos e privados do município responsáveis pelo tratamento de pessoas com transtornos mentais; formações focalizando a notificação das doenças mentais relacionadas ao trabalho no Sistema de Informação de Agravos de Notificação (Sinan) ${ }^{26}$; e confecção e envio de material educativo sobre a relação entre o trabalho e o adoecimento psíquico para os diversos equipamentos de saúde da cidade.

Essas práticas representam importantes ações de Educação Permanente para os profissionais da rede pública de saúde e buscam irradiar a discussão sobre a relação entre adoecimento psíquico e situações de trabalho na atualidade.

\section{Importância dos coletivos de resistências e os desafios vivenciados no SUS}

A ideia de que o trabalhador deve ser protagonista no enfrentamento do adoecimento mental relacionado ao trabalho esteve presente nas falas de todos os entrevistados. Ainda que nenhum deles tenha feito referência a algum modelo específico, os relatos sobre suas práticas indicam que eles consideram os pressupostos do Modelo Operário Italiano ${ }^{13}$, que tem como princípio a valorização do saber e da participação ativa dos trabalhadores na transformação da realidade de trabalho. Conforme defendido por Laurell e Noriega ${ }^{27}$, a grande inovação desse modelo reside no fato de que o sujeito condutor do processo de investigação da relação entre trabalho e saúde não é um especialista na área e, sim, os próprios trabalhadores, que possuem a vivência da realidade do ambiente de trabalho e o conhecimento empírico de suas reais condições.

Também Sato ${ }^{28}$, ao abordar o tema da vigilância em saúde nos ambientes de trabalho, afirma que o conhecimento prático dos trabalhadores deve ser considerado essencial para a definição do que é problema de saúde, para a elaboração de procedimento de identificação desses problemas e para a adoção de estratégias de intervenção que visem à melhoria das situações de trabalho.

Conforme destacado por Seligmann-Silva ${ }^{4}$, as resistências às pressões organizacionais e o combate à exploração e ao adoecimento mental provocado pelo trabalho na atualidade dificilmente poderão acontecer de forma solitária. Por esse motivo, a difusão da compreensão de que o adoecimento mental pode ser causado ou agravado por elementos relacionados ao trabalho e a percepção de que os trabalhadores não estão sozinhos pode ajudá-los a buscar o enfrentamento coletivo das condições precárias encontradas em seu cotidiano de trabalho. 
As práxis discutidas neste artigo revelam que as ações realizadas no âmbito do SUS podem contribuir com a defesa da saúde mental dos trabalhadores. No entanto, observamos as grandes dificuldades vivenciadas pelo sistema de saúde brasileiro e a intensificação de seu processo de desmonte, que cada vez mais incorpora a lógica do capitalismo organizacional em seu funcionamento ${ }^{29}$. Com a justificativa de intensa crise econômica e social, há o aumento das pautas privatistas no Brasil, o estímulo à terceirização da mão de obra e adoção de medidas inconsequentes de diminuição dos gastos públicos, fatores que representam grandes ameaças ao SUS. Nesse cenário, os profissionais da área da saúde enfrentam a degradação de suas próprias condições de trabalho, convivendo com falta de estrutura física, de pessoal e sendo desvalorizados socialmente.

Contudo, mesmo diante das grandes incertezas e desesperanças presentes neste momento da história do nosso país, a análise dos posicionamentos apresentados pelos participantes da pesquisa demonstra que eles possuem consciência do papel que desempenham no SUS, dos desafios encontrados neste contexto e revelam posturas éticas e políticas, materializadas em seus cotidianos de trabalho. Contribuem, desse modo, para manter vivos os valores que regem o SUS, proporcionando a atenção integral à saúde da população e procurando disseminar uma compreensão crítica da relação existente entre trabalho e subjetividade humana. Posicionamentos esses que combatem o fatalismo e a visão naturalizante $^{4,14}$ e individualizante do adoecimento/sofrimento mental relacionado ao trabalho, bem como favorecem o desenvolvimento de consciência e da organização coletiva dos trabalhadores. Por isso merecem reconhecimento e incentivo.

\section{Considerações finais}

As discussões realizadas neste artigo revelam que, apesar das inúmeras contradições que cercam a saúde pública brasileira, é possível encontrar práxis que possuem como origem o olhar crítico sobre a complexa conjuntura contemporânea e os diversos elementos que compõem a vida cotidiana dos indivíduos e afetam sua subjetividade. Elas partem de uma ampla compreensão do contexto social e de trabalho para o entendimento do processo saúde/adoecimento mental dos trabalhadores.
O esforço para colaborar com a informação e favorecer a tomada de consciência de diferentes atores sociais sobre os fatores existentes na organização do trabalho que podem conduzir ao adoecimento mental pôde ser verificado em diversas ações. Estas vão do atendimento sensível e integral dos trabalhadores que já apresentam sintomas de agravos da saúde mental, passam pelo incentivo à organização coletiva e chegam a ações de vigilância e fiscalização nos ambientes de trabalho e matriciamento dos demais equipamentos do SUS.

As práxis aqui apresentadas indicam que os participantes buscam colaborar com a mudança da realidade de trabalhadores adoecidos mentalmente e procuram atuar na prevenção de novos casos, valorizando a participação dos trabalhadores e colaborando para a propagação dessa consciência crítica da relação entre situação de trabalho e saúde mental.

É importante enfatizar, entretanto, que a intenção do estudo não foi atribuir exclusivamente aos profissionais da saúde a responsabilidade pelo combate ao adoecimento mental relacionado ao trabalho. $\mathrm{O}$ objetivo, vale repetir, foi o de analisar, valorizar e divulgar as diversas práticas comprometidas com os interesses da classe trabalhadora que são adotadas pelos entrevistados da pesquisa que, mesmo em espaços rodeados por dificuldades e incertezas, conseguem pensar e agir coletivamente.

Acreditamos que se faz necessário pensar em uma maior articulação entre os movimentos sociais, sindicais e a sociedade civil com a finalidade de discutir de forma mais detalhada essas práxis e procurar ampliá-las. Também deve-se incentivar a formação crítica e socialmente contextualizada dos profissionais da saúde e buscar outras ações que reivindiquem o fortalecimento de políticas públicas que visem à proteção da saúde integral dos trabalhadores.

Dessa maneira, consideramos que é preciso realizar amplos debates com o objetivo de barrar o aumento do sofrimento e adoecimento mental dos trabalhadores e sua naturalização. Nesse ponto, certamente, as práxis dos profissionais que participaram da pesquisa relatada aqui são inspiradoras, pois nos remetem ao engajamento ético-político com os princípios do SUS, ao combate do fatalismo, ao incentivo do protagonismo dos sujeitos e à esperança, à criatividade, à militância, características que estiveram na base da construção da reforma sanitária brasileira e, neste momento de nossa história, são essenciais para a preservação da saúde e dignidade da classe trabalhadora.

\section{Contribuição de autoria}

Ambas as autoras contribuíram para a estruturação, análise e interpretação de dados, redação e revisão do artigo e são responsáveis por todos os aspectos éticos e pelo conteúdo do trabalho. 


\section{Referências}

1. Organisation for Economic Cooperation and Development. Fit mind, fit job: from evidence to practice in mental health and work [Internet]. Paris: OECD Publishing; 2015 [acesso em 5 ago 2016]. Disponível em: http://www.oecd.org/els/fitmind-fit-job-9789264228283-en.htm

2. World Health Organization, International Labour Organisation. Mental health and work: impact, issues and good practices [Internet]. Geneva: WHO; 2000 [acesso em 16 jul 2016]. Disponível em: http://www.who.int/mental_health/media/en/712.pdf

3. Brasil. Ministério da Previdência Social. Cai número de acidentes de trabalho e aumenta afastamentos por transtornos mentais: previdência em questão [Internet]. Brasília, DF: Informativo Eletrônico do Ministério da Previdência Social; 2012 [acesso em 15 ago 2016]. Disponível em: http://www.previdencia. gov.br/arquivos/office/4_120326-105114-231.pdf

4. Seligmann-Silva E. Trabalho e desgaste mental: o direito de ser dono de si mesmo. São Paulo: Cortez; 2011.

5. Bernardo MH. Trabalho duro, discurso flexível: uma análise das contradições do toyotismo a partir da vivência de trabalhadores. São Paulo: Expressão popular; 2009.

6. Praun L. Reestruturação produtiva, saúde e degradação do trabalho. Campinas: Papel social; 2016.

7. Gaulejac V. Gestão como doença social: ideologia, poder gerencialista e fragmentação social. São Paulo: Ideias e letras; 2007.

8. Pina JA, Stotz EM. Intensificação do trabalho e saúde do trabalhador: uma abordagem teórica. Rev Bras Saude Ocup. 2014;39(130):150-60.

9. Bernardo $\mathrm{MH}$, Garbin AC. A atenção à saúde mental relacionada ao trabalho no SUS: desafios e possibilidades. Rev Bras Saude Ocup. 2011;36(123):103-17.

10. Campos GWS. O SUS entre a tradição dos Sistemas Nacionais e o modo liberal-privado para organizar o cuidado à saúde. Ciencia Saude Colet. 2007;12:1865-74.

11. Vázquez AS. Filosofia da praxis. Rio de Janeiro: Paz e Terra; 1977.

12. Minayo MCS. O desafio do conhecimento: pesquisa qualitativa em saúde. São Paulo: Hucitec; 1994.

13. Oddone I, Marri G, Gloria S, Briante G, Chiattella M, Re A. Ambiente do trabalho - a luta dos trabalhadores pela saúde. São Paulo: Hucitec; 1986.

14. Martín-Baró I. Psicología política latinoamericana. Caracas: Panapo; 1987.

15. Duarte J. Entrevista em profundidade. In: Duarte J, Barros A, organizadores. Métodos e técnicas de pesquisa em comunicação. São Paulo: Atlas; 2006. p. 62-83.

16. Maeno M, Paparelli R. Inovação para desenvolvimento de organizações sustentáveis: trabalho, fatores psicossociais e ambiente saudável.
Campinas: Centro de Tecnologia da Informação Renato Archer; 2013.

17. Brasil. Constituição da República Federativa do Brasil de 1988. Brasília, DF: Gráfica do Senado Federal; 1988.

18. Paparelli R, Sato L, Oliveira F. A saúde mental relacionada ao trabalho e os desafios aos profissionais da saúde. Rev Bras Saude Ocup. 2006;36(123):118-27.

19. Imbrizi JM, Keppler ILS, Vilhanueva MS. Grupo dos novos: relato de uma experiência de estágio com grupos de acolhimento de trabalhadores em um Centro de Referência em Saúde do Trabalhador (Cerest). Rev Bras Saude Ocup. 2013;38(128):302-14.

20. Andrade MC, Burali MAM, Vida A, Fransozio MBB, Santos RZ. Loucura e trabalho no encontro entre saúde mental e economia solidária. Psicol Ciencia Profissao. 2013;33(1):174-91.

21. Brasil. Ministério da Saúde. Secretaria de Vigilância à Saúde. Secretaria de Atenção à Saúde. Diretrizes Nacionais da Vigilância em Saúde. Brasília, DF: Ministério da Saúde; 2010.

22. Brasil. Ministério da Saúde. Núcleo Técnico da Política Nacional de Humanização. Humaniza SUS: equipe de referência e apoio matricial. Brasília, DF: Ministério da Saúde; 2004.

23. Santos APL, Lacaz FAC. Apoio matricial em Saúde do Trabalhador: tecendo redes na Atenção Básica do SUS, o caso de Amparo/SP. Ciencia Saude Colet. 2012;17(5):1143-50.

24. Dias MDA, Bertolini GCS, Pimenta AL. Saúde do trabalhador na atenção básica: análise a partir de uma experiência municipal. Trab Educ Saude. 2011;9(1):137-48.

25. Rigotti DG. Matriciamento e coprodução de autonomia: percepções dos apoiadores matriciais do SUS-Campinas [dissertação]. Campinas: Faculdade de Ciências Médicas, Universidade Estadual de Campinas; 2016.

26. Brasil. Ministério da Saúde. Portaria $\mathrm{n}^{\circ} 1.271$, de 6 de junho de 2014. Define a Lista Nacional de Notificação Compulsória de doenças, agravos e eventos de saúde pública nos serviços de saúde públicos e privados em todo o território nacional, nos termos do anexo, e dá outras providências. Diário Oficial da União. 9 jul 2014. Seção 1:67.

27. Laurell AC, Noriega, M. Processo de produção e saúde trabalho e desgaste operário. São Paulo: Hucitec; 1989.

28. Sato L. As implicações do conhecimento prático para a vigilância em saúde do trabalhador. Cad Saude Publica. 1996;12(4):489-95.

29. Blanch-Ribas JM. El capitalismo organizacional como factor de riesgo psicosocial: efectos psicológicos colaterales de las nuevas condiciones de trabajo dictadas por el capitalismo académico y sanitario implantado en universidades y hospitales no lucrativos de la red pública. Madrid: Ministerio Educación y Ciência de España; 2007. 